\title{
Efeitos da auriculoterapia com sementes de mostarda na dor lombar crônica de profissionais de enfermagem
}

\author{
Effects of auriculotherapy with mustard seeds on chronic lumbar pain of nursing professionals \\ Efectos de la auriculoterapia con semillas de mostaza en el dolor lumbar crónico de los \\ profesionales de enfermería
}

Ana Paula Gomes da Silva', Maria das Graças Rodrigues de Araújo², Marcelo Renato Guerino³

RESUMO | Mais de 60\% dos trabalhadores de enfermagem apresentam episódio de lombalgia durante um ano, provocando um impacto socioeconômico negativo, uma vez que é uma das maiores causas de faltas e afastamentos do trabalho. A auriculoterapia é uma técnica da acupuntura de baixo custo e não invasiva, que utiliza o pavilhão auricular como um microssistema do organismo humano mapeado por pontos que, estimulados, podem tratar diversas enfermidades. Sendo assim, o objetivo deste estudo foi verificar a eficácia da auriculoterapia com sementes de mostarda (Brassica juncea) na melhora da dor, na funcionalidade e na mobilidade lombar de profissionais técnicos e auxiliares de enfermagem do sexo feminino com dor lombar crônica. Foi realizado um ensaio clínico randomizado cego, dividido em dois grupos: um utilizou sementes de mostarda para acupressão nos pontos auriculares "Shen-Men", "Rim", "Simpático" e "Coluna Lombar" e o grupo placebo utilizou espuma de poliuretano de baixa densidade no lugar das sementes. Cada grupo realizou quatro sessões de auriculoterapia, uma vez por semana. Os grupos foram analisados por meio de termogramas infravermelhos e algometria por pressão para a dor, a medida dedo-chão para mobilidade e Questionário Roland-Morris para funcionalidade da coluna, com intervalo de confiança de 95\%. A auriculoterapia com sementes de mostarda reduziu a temperatura média nos termogramas analisados em $0,8^{\circ} \mathrm{C}$, bem como, aumentou o limiar de dor à pressão na coluna lombar das voluntárias em 0,4 Kgf, o que demonstra uma melhora significativa da dor lombar. Portanto, a auriculoterapia com sementes de mostarda mostrou-se eficaz na melhora da dor lombar. Descritores | Dor Lombar; Auriculoterapia; Auxiliares de Enfermagem.

ABSTRACT I More than $60 \%$ of nursing workers have an episode of low back pain for one year, causing a negative socioeconomic impact, as this is one of the major causes of absence from work. Auriculotherapy is a low-cost, noninvasive acupuncture technique that utilizes the auricular pavillion as a microsystem of the human organism mapped by points that when stimulated can treat various ailments. Thus, the aim of this study was to verify the efficacy of mustard seed auriculotherapy (Brassica juncea) in improving pain, functionality and lumbar mobility of female technical professionals and nursing assistants with chronic low back pain. A blind randomized clinical trial was conducted, divided into two groups: one used mustard seeds for acupressure in the ear points "Shen-Men", "Kidney", "Sympathetic" and "Lumbar Spine" and the other placebo group used low density polyurethane foam in place of seeds. Both groups performed four auriculotherapy sessions, one per week. The groups were analyzed using infrared thermograms and pressure algometry for pain, finger-floor measurement for mobility and Roland-Morris Questionnaire for spine functionality, with a 95\% confidence interval. Mustard seed auriculotherapy reduced the average temperature in the

\footnotetext{
'Universidade Federal de Pernambuco (UFPE) - Recife (PE), Brasil. E-mail: anap84@gmail.com. Orcid: 0000-0002-8791-212X ${ }^{2}$ Universidade Federal de Pernambuco (UFPE) - Recife (PE), Brasil. E-mail: mgrodriguesraujo@hotmail.com. Orcid: 0000-0002-9980-6172

3Universidade Federal de Pernambuco (UFPE) - Recife (PE), Brasil. E-mail: marceloguerino@hotmail.com. Orcid: 0000-0002-3439-9166
} 
thermograms analyzed by $0.8^{\circ} \mathrm{C}$, as well as increased the pain threshold to lumbar spine pressure of the volunteers by $0.4 \mathrm{Kgf}$ which shows a significant improvement of low back pain. Therefore, mustard seed auriculotherapy was effective in improving low back pain.

Keywords | Low Back Pain; Auriculotherapy; Nursing Professionals.

RESUMEN | Más del 60\% de los trabajadores de enfermería tienen un episodio de dolor lumbar durante un año, causando un impacto socioeconómico negativo, ya que es una de las mayores causas de ausencias y despidos del trabajo. La auriculoterapia es una técnica de acupuntura de bajo costo y no invasiva que utiliza el pabellón auricular como un microsistema del organismo humano mapeado por puntos que, estimulados, pueden tratar diversas enfermedades. Así, el objetivo de este estudio fue verificar la eficacia de la auriculoterapia con semillas de mostaza (Brassica juncea) en la mejora del dolor, la funcionalidad y la movilidad lumbar de mujeres profesionales técnicas y auxiliares de enfermería con dolor lumbar crónico. Se realizó un ensayo clínico aleatorizado ciego, dividido en dos grupos: uno utilizó semillas de mostaza para acupresión en los puntos auriculares "Shen-Men”, “Riñón”, "Simpático” y "Columna Lumbar" y el grupo placebo utilizó espuma de poliuretano de baja densidad en lugar de las semillas. Cada grupo realizó cuatro sesiones de auriculoterapia una vez por semana. Los grupos se analizaron mediante termogramas infrarrojos y algometría de presión para el dolor, prueba de distancia dedos-suelo para la movilidad y Cuestionario Roland-Morris para la funcionalidad de la columna vertebral, con un intervalo de confianza del 95\%. La auriculoterapia con semillas de mostaza redujo la temperatura promedio en los termogramas analizados en $0,8^{\circ} \mathrm{C}$, así como aumentó el umbral de dolor a presión en la columna lumbar de las voluntarias en 0,4 Kgf, lo que demuestra una mejora significativa en el dolor lumbar. Por lo tanto, la auriculoterapia con semillas de mostaza ha demostrado ser eficaz para mejorar el dolor lumbar.

Palabras clave | Dolor de la Región Lumbar; Auriculoterapia; Auxiliares de Enfermería.

\section{INTRODUÇÃO}

A dor lombar é um distúrbio musculoesquelético que interfere diretamente na qualidade de vida no trabalho, sobrecarregando o sistema de saúde por conta dos altos índices de absenteísmo e afastamentos ${ }^{1}$, um problema muito prevalente nos profissionais da enfermagem devido à natureza de suas atividades laborais. Existem diversas abordagens não medicamentosas para o manejo da dor lombar, como exercícios físicos de fortalecimento e alongamento muscular, terapia manual, laserterapia de baixa potência, educação do paciente sobre o problema, programa de reabilitação multidisciplinar biopsicossocial, terapia cognitivo-comportamental e, dentro desse universo, temos a auriculoterapia, uma técnica da acupuntura que utiliza o pavilhão auricular para efetuar o tratamento de diversas enfermidades, trabalha com pontos situados na orelha que compreendem um microssistema do organismo humano, ou seja, a representação de todo o corpo está contida no pavilhão auricular².

Os materiais mais utilizados na auriculoterapia são as agulhas semipermanentes e as sementes de mostarda, que são fixadas na orelha por meio de adesivos, geralmente por um período mínimo de quatro dias, até o máximo de uma semana ${ }^{3}$.

O alívio da dor pela auriculoterapia também é explicado pela liberação de neurotransmissores que a aplicação nos pontos proporciona. $\mathrm{O}$ estímulo realizado num ponto de acupuntura promove resposta neuro-humoral do organismo, o que faz com que as células secretem substâncias opioides como a endorfina, serotonina e encefalina, que são analgésicos naturais que propiciam o alívio de dores e a sensação de bem-estar ${ }^{4}$.

O presente estudo teve por objetivo investigar a auriculoterapia com sementes de mostarda (Brassica juncea) como um possível recurso terapêutico para a melhora da dor à pressão, da funcionalidade e aumento na mobilidade da coluna lombar e suas possíveis alterações termográficas em profissionais técnicos e auxiliares de enfermagem com dor lombar crônica.

\section{METODOLOGIA}

Este estudo é um ensaio clínico randomizado por "intenção de tratar" com cegamento simples da amostra, seguindo o check-list do Consolidated Standards of Reporting Trials - CONSORT, de 2010, na extensão não farmacológica. As voluntárias foram devidamente esclarecidas sobre os procedimentos utilizados por meio do Termo de Consentimento Livre e Esclarecido (TCLE), sendo dessa maneira obtida a sua autorização para realização do estudo. 
As técnicas e auxiliares de enfermagem do Hospital das Clínicas da Universidade Federal de Pernambuco (HCUFPE) e do Hospital Getúlio Vargas (HGV), ambos situados em Recife, foram analisadas no Laboratório de Cinesioterapia e Recursos Terapêuticos Manuais (LACIRTEM) do Departamento de Fisioterapia da Universidade Federal de Pernambuco.

Foram analisados 23 profissionais de enfermagem do sexo feminino que afirmaram sentir dor lombar há pelo menos três meses e que trabalham nas enfermarias de adultos, prestando assistência de enfermagem direta aos pacientes internados.

Foi realizado cálculo amostral pelo software $\mathrm{G}^{*}$ Power 3.1.9.2, considerando o teste de Wilcoxon, também conhecido como teste de Mann-Whitney, para a comparação dos resultados das avaliações entre os grupos "auriculoterapia" e "placebo", considerando nível de significância $(\alpha)$ de 0,05 .

O tamanho do efeito foi calculado considerando uma diferença na média entre os grupos de $0,6 \mathrm{~kg} / \mathrm{cm}^{2}$ e desvio padrão de $0,5 \mathrm{~kg} / \mathrm{cm}^{2}$ para a algometria; uma diferença na média entre os grupos de $0,6^{\circ} \mathrm{C}$ e desvio padrão de $0,5^{\circ} \mathrm{C}$ para a termografia; e uma diferença na média entre os grupos de seis pontos e desvio padrão de cinco pontos para o questionário Roland Morris, todas baseadas nos resultados dos trabalhos de Hakgüder et al. ${ }^{5}$ e Imamura et al. ${ }^{6}$.

Assim, o tamanho amostral necessário foi estimado em 20 pacientes (poder $=0,80 ;=0,05 ;$ tamanho do efeito $=1,20$ ), sendo 10 do grupo intervenção e 10 do grupo placebo. Foi realizada a comparação entre os grupos através da "variação" da melhora, sendo calculado da seguinte forma: Valor_pós_tto - Valor_pré_tto=valor de melhora (ou $\Delta$ tto), onde "tto" significa "tratamento" na fórmula utilizada, indicando o quanto a paciente melhorou ou piorou em cada grupo e comparando essa melhora ou piora entre os grupos.

Os critérios de inclusão foram profissionais técnicos e auxiliares de enfermagem do sexo feminino das enfermarias de adultos do HC-UFPE e do HGV que prestam cuidados diretos aos pacientes internados, com IMC entre 18,5 e 29,9, faixa etária entre 20 e 60 anos, portadores de lombalgia crônica inespecífica ( $\geq 03$ meses) sem diagnóstico prévio autodeclarado de hérnia discal, tumor maligno de qualquer tipo, doença degenerativa, infecciosa ou reumática (artrite reumatoide, espondilite anquilosante e osteoartrose) ou cirurgia prévia na coluna lombar. Como critérios de exclusão foram gestantes e lactantes, alérgicas a esparadrapo micropore, profissionais que exercem apenas atividades burocráticas que não o cuidado direto aos pacientes internados.

As participantes foram distribuídas por meio de aleatorização simples computadorizada (http:// randomization.com) em dois grupos: Grupo 1 Auriculoterapia, que recebeu tratamento com sementes de mostarda que estimularam os pontos terapêuticos do protocolo, e Grupo 2 - Placebo, que recebeu tratamento com espuma convencional de poliuretano de densidade $26 \mathrm{~kg} / \mathrm{m} 3$ no lugar das sementes de mostarda nos pontos do protocolo para a dor lombar. A espuma por ser flexível e de baixa densidade não fez a estimulação mecânica dos pontos auriculares.

O cegamento foi possível devido à diferença entre a semente e a espuma não ser perceptível pelas voluntárias, ficando apenas a pesquisadora ciente dessa diferença. Nenhuma das participantes havia feito tratamento prévio com auriculoterapia e os grupos não tinham contato entre si.

Primeiramente, foram coletadas as informações de caracterização da amostra e assinatura do Termo de Consentimento Livre e Esclarecido Em seguida, foi aplicado o Questionário de Incapacidade Roland-Morris para dor lombar validado para o $\mathrm{Brasil}^{7}$ nas participantes que não apresentassem nenhum critério de exclusão.

$\mathrm{Na}$ semana seguinte, as participantes foram ao Laboratório de Cinesioterapia e Recursos Terapêuticos Manuais (LACIRTEM) do Departamento de Fisioterapia da UFPE, onde foi realizado o exame termográfico com câmera termográfica (FLIR Systems - E40, Suécia), com sensibilidade de $0,05^{\circ} \mathrm{C}$, e resolução infravermelha de 320 x 240 pixels, programada com emissividade de 0,98 , temperatura refletida de $20^{\circ} \mathrm{C}$, fusão imagem na imagem e paleta arco-íris.

Durante a mensuração, as participantes ficaram em pé com o tronco ereto, sobre superfície emborrachada isolante. A região analisada foi mantida livre de roupas. A imagem foi capturada a uma distância de $100 \mathrm{~cm}$ da região a ser analisada para permitir o enquadramento adequado, contra anteparo de cor preta para evitar a reflexão da radiação infravermelha.

A região examinada foi delimitada de acordo com o protocolo Glamorgan ${ }^{8}$ para a lombar, onde as temperaturas mínimas, médias e máximas da região de interesse foram calculadas por software FLIR Quick Report (versão 1.2). As voluntárias permaneceram na posição solicitada por 10 minutos para aclimatação da câmera, com temperatura ambiente de 22 a $24^{\circ} \mathrm{C}$, controlada por condicionadores de ar e iluminada com luzes fluorescentes frias, sem a 
presença de equipamentos elétricos geradores de calor ou a incidência direta de luz solar.

A temperatura e umidade foram monitoradas por termo-higrômetro digital marca Incoterm, modelo 7666.02.0.00, com temperatura em graus Celsius e umidade relativa do ar em porcentagem que oscilou entre 60 e $65 \%$. As participantes foram instruídas a evitar álcool, tabaco, exercício físico, banho, ingestão de substâncias estimulantes como a cafeína por ao menos duas horas antes do exame.

Para avaliação da dor, as participantes foram posicionadas sentadas em banco sem encosto, joelhos fletidos a $90^{\circ}$, cabeça em posição neutra e ombros relaxados. Foi pedido que a voluntária indicasse com o dedo o ponto mais dolorido e, em seguida, realizou-se palpação da musculatura da região lombar para determinação e averiguação dos pontosgatilho. A área foi, então, marcada com caneta esferográfica para aferição, sendo fotografada em câmera digital para a reavaliação após as sessões de auriculoterapia.

Para mensuração do limiar de dor à pressão foi utilizado algômetro de pressão (AP), marca Kratos, modelo DDK eletrônico, com uma sonda metálica, plana e circular, medindo $1 \mathrm{~cm}$ de diâmetro, pressão em kgf. As voluntárias sentadas (posição que facilita a aplicação perpendicular da pressão com o aparelho nos pontos-gatilhos) foram instruídas sobre a aplicação do AP no ponto-gatilho marcado e orientadas a dizerem "pare" imediatamente quando sentissem dor. Cada medida foi realizada duas vezes e considerada a média entre elas.

Para avaliação da mobilidade lombar foi realizada a Medida Dedo-Chão, ou Finger-Floor Distance (FFD), onde as participantes foram orientadas a realizarem uma flexão anterior do tronco até o primeiro ponto de dor ou resistência com ambas as mãos unidas em direção ao chão. A distância da ponta do terceiro dedo para o chão foi medida em centímetros $(\mathrm{cm})$ com régua de acrílico escolar graduada. Foram tomadas duas medidas e considerada a média entre elas. Os protocolos de tratamento utilizados nos grupos foram realizados em quatro sessões que ocorreram uma vez por semana, respeitando a escala de trabalho dos participantes.

A intervenção utilizada foi a acupressão auricular com sementes de mostarda, por serem esféricas e rígidas, com cerca de $2 \mathrm{~mm}$ de diâmetro, de baixo custo e orgânicas, produzindo ação mecânica sobre os pontos terapêuticos da orelha para dor lombar. A espuma de poliuretano foi utilizada no lugar das sementes para o grupo placebo, não estimulando os pontos do protocolo por serem flexíveis.
Tanto as sementes como as espumas foram presas à orelha das participantes por esparadrapo micropore.

As sementes e espumas foram colocadas sobre os pontos com auxílio de uma pinça de dissecção, após limpeza de toda a região auricular com álcool gel a $70 \%$. As participantes não tiveram contato entre si ou entre os grupos e não tinham feito nenhum tratamento prévio com a auriculoterapia.

Os pontos escolhidos foram direcionados segundo os descritos na Medicina Tradicional Chinesa (MTC): "Shen Men", "Rim”, "Simpático" e "Coluna Lombar". O último ponto foi estimulado com duas sementes, acompanhando o desenho da própria coluna lombar na orelha. A escolha dos pontos foi baseada em trabalhos sobre a auriculoterapia como os de Santos ${ }^{9}$ e Souza ${ }^{10}$, que citam a mesma cartografia para a melhora de quadros álgicos lombares.

As participantes foram orientadas a estimularem as sementes, de forma suave, sobre os pontos em média três vezes ao dia (ao levantar-se, no meio da tarde e antes de dormir), com duração de um minuto em cada ponto. As sementes permaneceram na orelha até a sessão seguinte.

Cada sessão foi iniciada pela orelha do lado dominante da participante (destro ou canhoto). A cada sessão semanal houve alternância entre orelhas direita e esquerda para evitar a saturação dos pontos até o final do ciclo de tratamento.

Todas as participantes foram acionadas por aplicativo de mensagens instantâneas de celular como forma de reforçar as orientações para a auriculoterapia e avaliações da região lombar. Apenas três participantes relataram queda das sementes/espuma sendo recolocadas pela pesquisadora em menos de 12 horas.

Uma semana após o fim das sessões, foi realizada reavaliação da região lombar das voluntárias que não apresentaram nenhum critério de exclusão para análise dos objetivos do estudo. Durante o período de estudo, as participantes foram orientadas a não utilizarem qualquer medicação (analgésico, anti-inflamatório e/ou relaxante muscular), bem como a não se submeterem a outro tipo de tratamento. Todas as sessões foram realizadas nos locais de trabalho das voluntárias, com exceção de duas delas, que precisaram que se realizasse em suas residências, devido à troca de plantão e início de férias. A pesquisadora arcou com os custos de deslocamento.

\section{Análise estatística}

Para análise de distribuição dos dados foi realizado o teste de normalidade Shapiro-Wilk, utilizando o teste $\mathrm{t}$ 
de Student ou teste de Mann-Whitney para comparação das médias entre os grupos placebo e auriculoterapia. Para comparação das variáveis categóricas foi realizado o teste de Qui-quadrado ou o teste Exato de Fisher. As variáveis contínuas foram expressas em média e desvio padrão, diferença de média e intervalo de confiança de $95 \%$, ou mediana e intervalo quartil. As variáveis categóricas foram expressas em número de casos e frequência.

A análise estatística foi realizada com o Graph Pad Prism 4.0 (GraphPad Software Inc., USA) e Sigma Plot 12.0 (Systat Software, Inc., Germany). Considerou-se significativos valores de $\mathrm{p}<0,05$. Para as perdas imputou-se valores iguais aos da avaliação pré-auriculoterapia, tomando como o pior desfecho, sem melhora alguma, uma vez que a análise foi por intenção de tratar.

\section{RESULTADOS}

Conforme mostrado na Figura 1, das 54 participantes elegíveis, 31 foram excluídas pelo IMC ou por não realizarem os testes pré-intervenção, e 23 foram randomizadas em dois grupos, sendo 11 alocadas no grupo Placebo e 12 no grupo Auriculoterapia. Houve perda de seguimento de três participantes por não realizarem as quatro sessões de auriculoterapia, sendo uma por doença e outras duas por desistência.

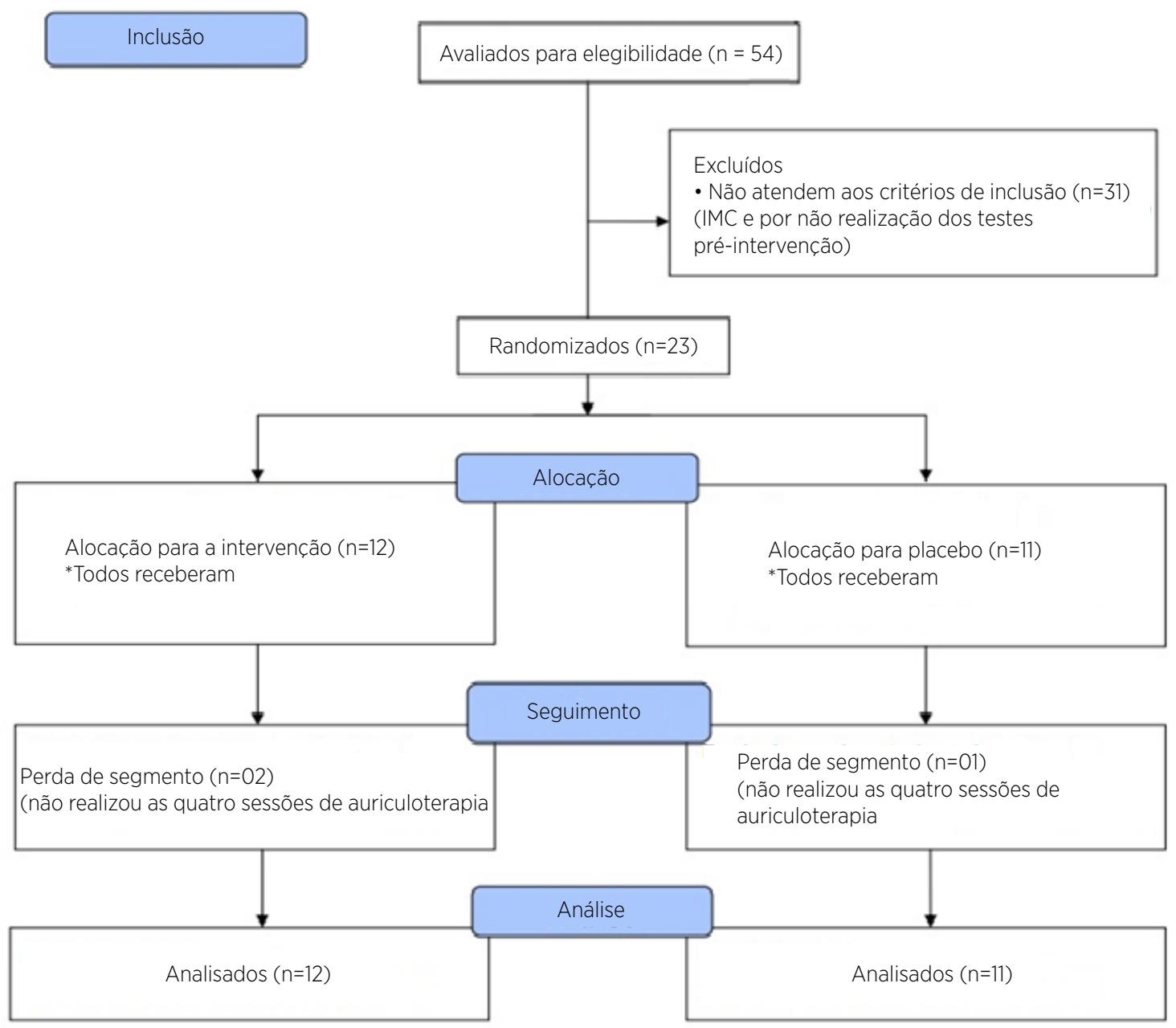

Figura 1. Fluxograma CONSORT

A Tabela 1 mostra os resultados dos grupos antes e depois das sessões de auriculoterapia para temperatura, limiar de dor à pressão, escore Roland-Morris e medida dedo-chão.

O grupo placebo partiu de uma média de 9,9 $\pm 4,6$ pontos do escore Roland-Morris, enquanto o grupo intervenção partiu de uma média de $9 \pm 4,5$ pontos. Já a variação para os grupos, após as sessões de auriculoterapia, foi de $1,4 \pm 5,2$ para o grupo placebo e de $1,8 \pm 2,4$ para o grupo intervenção, o que não mostrou diferença estatisticamente significativa. 
Tabela 1. Variáveis Pré e Pós-intervenção nos Grupos Placebo e Auriculoterapia.

\begin{tabular}{|c|c|c|c|c|c|c|c|c|}
\hline & \multicolumn{3}{|c|}{ Placebo } & \multicolumn{3}{|c|}{ Auriculoterapia } & \multirow{2}{*}{$\begin{array}{l}\text { Diff_média } \\
\text { (IC) } \\
\Delta \text { pré-pós }\end{array}$} & \multirow{2}{*}{$\begin{array}{c}\text { p-valor }{ }^{1} \\
\text { Entre grupos }\end{array}$} \\
\hline & Pré & Pós & $\Delta$ pré-pós & Pré & Pós & $\Delta$ pré-pós & & \\
\hline Q. Rolland-Morris & $9,9 \pm 4,6$ & $8,5 \pm 5,0$ & $-1,4 \pm 5,2$ & $9,0 \pm 4,5$ & $7,2 \pm 5,4$ & $-1,8 \pm 2,4$ & $-0,4(-3,9$ a 3,0$)$ & $0,778^{\#}$ \\
\hline Limiar de dor à pressão (Kgf) & $1,6 \pm 0,5$ & $1,4 \pm 0,5$ & $-0,2 \pm 0,7$ & $1,6 \pm 0,8$ & $2,0 \pm 1,2$ & $0,4 \pm 0,8$ & $0,6(0,1$ a 1,4$)$ & 0,038 \\
\hline Distância Dedo-Chão (cm) & $12,5 \pm 6,4$ & $10,2 \pm 5,8$ & $-2,3 \pm 3,1$ & $15,5 \pm 9,0$ & $13,1 \pm 8,8$ & $-2,4 \pm 3,5$ & $0,1(-2,8$ a 2,9$)$ & 0,974 \\
\hline Temperatura $\left({ }^{\circ} \mathrm{C}\right)$ & $32,7 \pm 1,0$ & $32,9 \pm 0,6$ & $0,2 \pm 1,1$ & $33,2 \pm 0,5$ & $32,4 \pm 0,7$ & $-0,8 \pm 0,8$ & $-1,0(-0,5 a-2,1)$ & 0,003 \\
\hline
\end{tabular}

Inicialmente, os dois grupos apresentaram limiares baixos à pressão ao estímulo doloroso. $\mathrm{O}$ limiar de dor inicial em quilograma-força para ambos os grupos foi em média 1,6, com desvio-padrão de $\pm 0,5$ para o grupo placebo e $\pm 0,8$ para o grupo intervenção. Após as sessões de auriculoterapia foi observado um aumento no limiar de dor do grupo Auriculoterapia e uma discreta diminuição desse limiar no grupo Placebo estatisticamente significantes.

Os grupos não foram significativamente diferentes entre si para a medida dedo-chão, tanto pré quanto pósintervenção, e verificou-se mobilidade diminuída em ambos os grupos.

Quando observamos os valores de temperatura após as intervenções, eles aumentaram no grupo Placebo e diminuíram no grupo Auriculoterapia. Ambos os grupos mostravam termogramas assimétricos da coluna lombar, onde as áreas em branco sobre a grande área vermelha representam as maiores temperaturas de superfície da pele, sendo respectivas aos pontos de dor que relatavam (Figura 2).

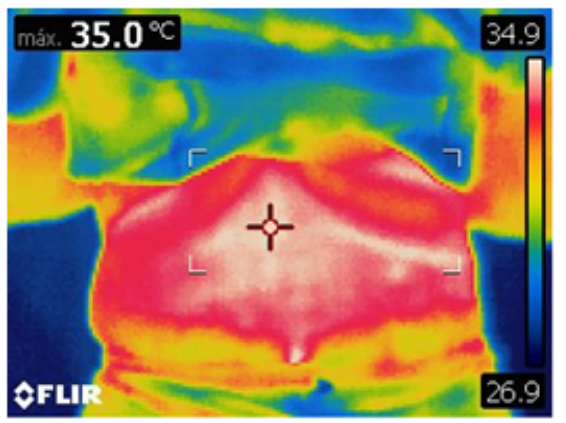

Participante do Grupo Placebo pré intervenção

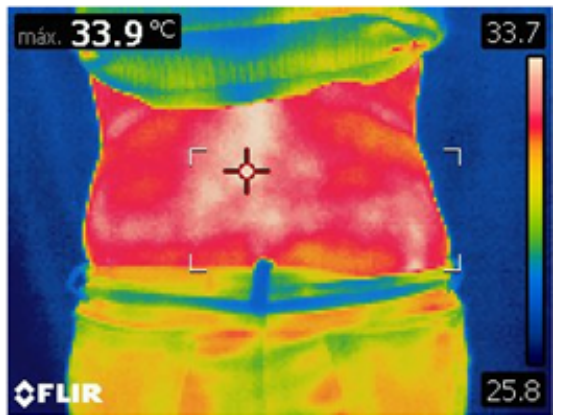

Participante do Grupo Auriculoterapia pré intervenção

Figura 2. Termogramas pré-intervenção, grupos Placebo e Auriculoterapia
Também visualizou-se variações nos termogramas finais, mostrando diminuição de área branca (Figura 3), quando comparados aos termogramas iniciais. $<$ figura $>$

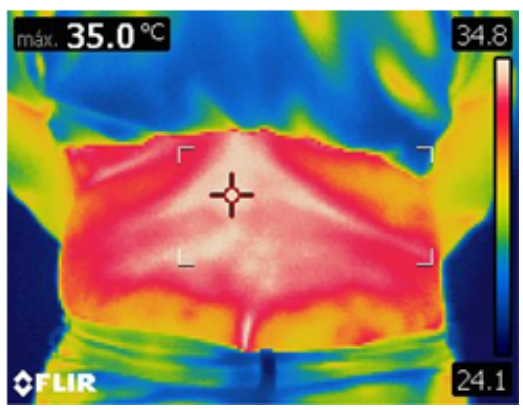

Participante do Grupo Placebo pós intervenção

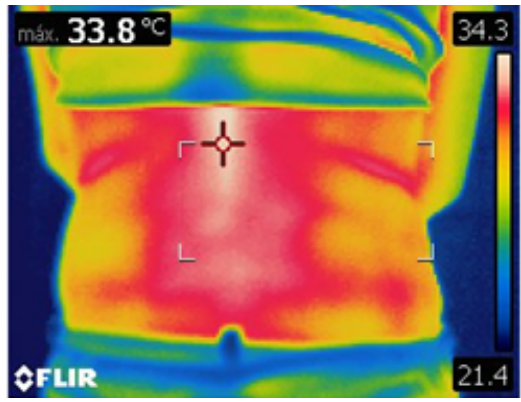

Participante do Grupo Auriculoterapia pós intervenção

Figura 3. Termogramas pós-intervenção, grupos Placebo e Auriculoterapia

\section{DISCUSSÃO E CONCLUSÕES}

A auriculoterapia com sementes de mostarda demonstra ser uma forma interessante de se promover tratamento, aumentando o limiar de dor à pressão na coluna lombar dos voluntários investigados neste estudo. A proporção encontrada para sobrepeso e obesidade (62\%) está acima do encontrado em estudos como o de Malta et al. ${ }^{11}$, que obteve $43,1 \%$ de sobrepeso e obesidade em uma população feminina entre 2006 e 2013, e de Citko et al. ${ }^{12}$, 48,44\% em enfermeiros poloneses. Neste estudo as participantes com obesidade foram excluídas.

Em relação aos efeitos promovidos pela auriculoterapia na funcionalidade lombar, medida por meio do escore de Roland-Morris, os resultados foram semelhantes entre os 
grupos, não apresentando diminuição significativa desse valor concomitante à melhora da dor, assemelhando-se ao observado nos trabalhos de Ushinohama et al. ${ }^{13}$ que utilizaram pelo menos um dos mesmos pontos auriculares utilizados em neste trabalho.

No estudo de Ushinohama et al. percebeu-se que a amostra estudada também não tinha valores médios de escore de Roland-Morris significativos para a incapacidade lombar (média de 4,2) assim como em neste estudo, embora a dor fosse presente e tenha sido melhorada com apenas uma sessão da intervenção. Da mesma forma, testaram o equilíbrio postural e não houve melhora do equilíbrio como houve com a dor lombar.Já no estudo de Moura et al. ${ }^{14}$, houve melhora no escore de incapacidade de Roland-Morris, porém, esse estudo avaliou da mesma forma a dor na coluna lombar, torácica e cervical, além da população ter sido mista (homens e mulheres) e portadora de osteoartrite, aplicando cinco sessões de auriculoterapia ao invés de quatro sessões, conforme a metodologia deste estudo.

Kim et al. ${ }^{15}$, ao aplicarem o questionário Roland-Morris em pacientes com dor lombar crônica e dor lombar com dor radicular, mostraram que o grupo com dor radicular apresentou escores maiores. Os pesquisadores aplicaram ainda questionários para avaliação psicológica dos grupos, o que evidenciou forte risco de comprometimento da saúde mental no grupo com maior dor e incapacidade lombar, fazendo com que os autores sugerissem aliar uma abordagem psicológica nos tratamentos da dor lombar crônica, buscando melhorar os escores de funcionalidade pelas características multifatoriais da dor.

Apesar dos resultados do questionário não serem estatisticamente diferentes entre os grupos do estudo, existe uma tendência de melhora clínica que não pode ser desprezada, corroborando o uso da terapêutica estudada para influência na funcionalidade lombar.

Imamura et al. ${ }^{6}$ constatou que pessoas saudáveis suportam em média 4,75kgf nos dermátomos da região lombar. Em nosso estudo, que comparou apenas indivíduos já portadores de dor lombar, encontramos média de 1,6kgf suportados pelas voluntárias na região lombar de ambos os grupos, mostrando que a dor lombar de fato diminui a quantidade de pressão suportada na região.

Achados semelhantes se mostraram no estudo de Ozdolap, Sarikaya e Kokturk ${ }^{16}$, em amostra composta por 132 pacientes, em que 70 relatavam dor lombar crônica e 62 eram indivíduos saudáveis, ambos os grupos com média de idade semelhante à da amostra deste trabalho, em que também demonstrou-se que o limiar de dor à pressão foi menor no grupo com dor lombar crônica, sendo menor ainda nas mulheres que compunham a amostra.

Em nosso estudo, a auriculoterapia fez com que o grupo de intervenção tivesse limiares de dor maiores em relação ao grupo placebo, resultados comparáveis aos de Yeh et al. ${ }^{17}$, que obtiveram melhora de $70 \%$ na dor medida em algômetro, eles também usaram sementes de mostarda como intervenção, mesmos pontos auriculares aplicados, além da população estudada por eles ser majoritariamente feminina.Além da dor lombar, os trabalhos de Santoro et al. ${ }^{18}$ mostram o algômetro como instrumento de aferição de limiares de dor à pressão pós-tratamento para dor de ombro e dos dedos, sendo mais uma vez as sementes de mostarda utilizadas para a acupressão auricular, com melhora da dor dos indivíduos estudados em ambos os estudos.

Embora a termografia infravermelha não meça diretamente a dor e sim as alterações vasomotoras da região analisada, em nosso estudo essa relação entre dor e imagem infravermelha ficou estabelecida, como nos mostra as alterações pré e pós-intervenção entre os grupos, medidas pelos termogramas (Figuras 2 e 3 ) e a variação dos limiares de dor à pressão, medidas pelo algômetro ${ }^{19}$. A termografia não deve ser utilizada isolada e sim em conjunto a outras avaliações clínicas para buscar esclarecimento diagnóstico e acompanhamento dos pacientes com dor.

No estudo de Chen e $\mathrm{Hu}^{20}$ as alterações termográficas em indivíduos com dor lombar foram compatíveis com hérnia de disco lombar, confirmadas por ressonância nuclear magnética e tomografia computadorizada em $66 \%$ dos casos. Em nosso estudo, não havia diagnóstico prévio da causa da dor lombar.

Ao revisar a literatura, Bardhan et al. ${ }^{21}$ mostrou que a termografia pode realizar essa mensuração da dor no futuro, quando forem desenvolvidos algoritmos capazes de quantificar a reação dolorosa uniformemente nos indivíduos com alterações térmicas nas mais diversas enfermidades. Como desafios estão a subjetividade da dor, o controle dos fatores ambientais durante a captura das imagens e a tecnologia das câmeras infravermelhas.

A mobilidade lombar, medida por meio da distância dedo-chão, se mostra diminuída em profissionais de enfermagem com dor lombar do sexo feminino, como mostrado no estudo de Takenaka et al. ${ }^{22}$, quando avaliaram enfermeiras japonesas não submetidas a tratamento. Encontramos o mesmo resultado neste estudo.O sedentarismo registrado entre as participantes do nosso estudo pode ter contribuído para a diminuição dessa mobilidade como é mostrado por Takenaka et al. ${ }^{22}$, 
que observaram a melhora da mobilidade lombar após programa de exercícios de três meses.

Ainda em nosso estudo, não houve diferença significante pré e pós-auriculoterapia entre os grupos estudados nas medidas de mobilidade lombar, semelhante ao estudo de Helmhout et al. ${ }^{23}$ que, em amostra de 141 participantes com dor lombar e tratados com fisioterapia, apresentou melhora da dor lombar, mas não da mobilidade, parâmetros que não necessariamente estão associados, embora a melhora da mobilidade com exercícios será maior em indivíduo sem dor.

Ekedahl, Jonsson e Frobell ${ }^{24}$ mostraram forte relação entre a medida dedo-chão e a funcionalidade lombar, medida pelo questionário Roland-Morris, o que também foi observado em nosso estudo. Tanto funcionalidade como mobilidade lombar não tiveram alterações significativas após a auriculoterapia, embora a dor tenha diminuído no grupo tratado.

A auriculoterapia vem sendo cada vez mais estudada, porém ainda faltam estudos conduzidos com amostras maiores e parâmetros melhor controlados. Zhao et al..$^{25}$ em revisão sistemática da literatura, buscando a eficácia da auriculoterapia para a dor lombar, encontrou dois estudos, dentre 15 trabalhos, utilizaram sementes de mostarda Nenhum desses trabalhos avaliou, conjuntamente à dor, a funcionalidade, a mobilidade ou termogramas infravermelhos.

Diante do exposto, o presente estudo mostrou que a auriculoterapia com sementes de mostarda para a dor lombar crônica em profissionais de enfermagem reduziu a temperatura média nos termogramas analisados e aumentou o limiar de dor à pressão em quilograma-força na coluna lombar das voluntárias de forma não invasiva e de baixo custo.

\section{AGRADECIMENTOS}

Nossos agradecimentos ao Laboratório de Cinesioterapia e Recursos Terapêuticos Manuais (LACIRTEM) da UFPE, que proporcionou o local de estudo deste trabalho.

\section{REFERÊNCIAS}

1. Ribeiro T, Serranheira F, Loureiro H. Work related musculoskeletal disorders in primary health care nurses. Appl Nurs Res. 2017;33:72-7. doi: 10.1016/j.apnr.2016.09.003.
2. Kurebayashi LFS, Silva MJP. Auriculoterapia Chinesa para melhoria de qualidade de vida de equipe de Enfermagem. Rev Bras Enferm. 2015;68(1):117-23. doi: 10.1590/0034-7167.2015680116p

3. Zumstein A. Apostila de Acupuntura Auricular. São Paulo: Curso de formação do Instituto Brasileiro de Acupuntura (IBRAM); 2012

4. Silvério-Lopes S. Analgesia por acupuntura. Curitiba: Omnipax; 2013.

5. Hakgüder A, Birtane M, Gürcan S, Kokino S, Turan FN. Efficacy of low level laser therapy in myofascial pain syndrome: An algometric and thermographic evaluation. Lasers Surg Med. 2003;33(5):339-43. doi: 10.1002/Ism.10241.

6. Imamura M, Chen J, Matsubayashi SR, Targino RA, Alfieri FM, Bueno DK, et al. Changes in Pressure Pain Threshold in Patients With Chronic Nonspecific Low Back Pain. Spine. 2013;38(24):2098107. doi: 10.1097/01.brs.0000435027.50317.d7.

7. Sardá Júnior JJ, Nicholas MK, Pimenta CAM, Asghari A, Thieme AL. Validation of the Roland Morris Disability Questionnaire for general pain. Rev Dor. 2010;11(1):28-36.8. Ammer K. The Glamorgan Protocol for recording and evaluation of thermal images of the human body. Thermol Int. 2008;18(4):124-9.9. Santos JF. Auriculoterapia e Cinco Elementos. 3a ed. São Paulo: Ícone; 2010.

10. Souza MP. Tratado de auriculoterapia. Brasília: Marcelo Pereira de Souza; 2007.

11. Malta DC, Santos MAS, Andrade SSCA, Oliveira TP, Stopa SR, Oliveira MM, et al. Tendência temporal dos indicadores de excesso de peso em adultos nas capitais brasileiras, 2006-2013. Ciênc Saude Colet. 2016;21(4):1061-9. doi: 10.1590/1413-81232015214.12292015.

12. Citko A, Górski S, Marcinowicz L, Górska A. Sedentary Lifestyle and Nonspecific Low Back Pain in Medical Personnel in North-East Poland. Biomed Res Int. 2018;2018:1965807 doi: 10.1155/2018/1965807.

13. Ushinohama A, Cunha BP, Costa LO, Barela AM, Freitas PB. Effect of a single session of ear acupuncture on pain intensity and postural control in individuals with chronic low back pain: a randomized controlled trial. Braz J Phys Ther. 2016;20(4):32835. doi: 10.1590/bjpt-rbf.2014.0158.

14. Moura CC, Junes DH, Ruginsk SG, Souza VHS, Assis BB, Chaves ECL. Action of ear acupuncture in people with chronic pain in the spinal column: a randomized clinical trial. Rev Lat Am Enfermagem. 2018;26:e3050. doi: 10.1590/1518-8345.2678.3050.

15. Kim S, Kim JH, Kim YL, Lee SM. Comparison of pain, disorder, back performance, and psychological factors in patients with low back pain and radicular pain. J Phys Ther Sci. 2018;30(1):12731. doi: 10.1589/jpts.30.127.

16. Ozdolap S, Sarikaya S, Kokturk F. Evaluation of Pain Pressure Threshold and Widespread Pain in Chronic Low Back Pain. Türk Fiz Tip Rehab Derg. 2014;60(1):32-6. doi: 10.5152/ tftrd.2014.71602.

17. Yeh CH, Chien LC, Balaban D, Sponberg R, Primavera J, Morone NE, et al. A Randomized Clinical Trial of Auricular Point Acupressure for Chronic Low Back Pain: A Feasibility Study. Evid Based Complement Alternat Med. 2013;2013:196978. doi: 10.1155/2013/196978.

18. Santoro A, Nori SL, Lorusso L, Secondulfo C, Monda M, Viggiano A. Auricular Acupressure Can Modulate Pain Threshold. Evid 
Based Complement Alternat Med. 2015;2015:457390. doi: 10.1155/2015/457390.

19. Brioschi ML, Abramavicus S, Correa CF. Valor da imagem infravermelha na avaliação da dor. Rev Dor. 2005;6(1):51424.20. Chen X, Hu Y. The Clinical Application of Infrared Thermal Imaging in Lumbocrural Pain. Curr Med Imaging Rev. 2018;14(5):818-21. doi: 10.2174/1573405613666170607150557.

21. Bardhan S, Bhowmik MK, Nath S, Bhattacharjee D. A review on inflammatory pain detection in human body through infrared image analysis. Proceedings of the International Symposium on Advanced Computing and Communication (ISACC); 2015 Sep 14-15; Silchar, India. New York: IEEE; 2015. doi: 10.1109/ isacc.2015.7377350.

22. Takenaka H, Nishihama K, Yaguchi A, Ushijima H, Miyachi Y, Furuta K, et al. Cross-sectional Study of Actual Conditions and
Related Factors of Low Back Pain among Hospital Workers. Rigakuryoho Kagaku. 2015;30(2):297-300. doi: 10.1589/ rika.30.297.

23. Helmhout PH, Witjes M, Nijhuis-Van Der Sanden RW, Bron C, van Aalst M, Staal JB. The effects of lumbar extensor strength on disability and mobility in patients with persistent low back pain. J Sports Med Phys Fitness. 2017;57(4):411-7. doi: 10.23736/ S0022-4707.16.06270-8

24. Ekedahl H, Jönsson B, Frobell RB. Fingertip-to-Floor Test and Straight Leg Raising Test: Validity, Responsiveness, and Predictive Value in Patients With Acute/Subacute Low Back Pain. Arch Phys Med Rehabil. 2012;93(12):2210-5. doi: 10.1016/j.apmr.2012.04.020.

25. Zhao H, Tan JY, Wang T, Jin L. Auricular therapy for chronic pain management in adults: A synthesis of evidence. Complement Ther Clin Pract. 2015;21(2):68-78. doi: 10.1016/j.ctcp.2015.03.006. 